\title{
ISAR Imaging of Ship Target with Complex Motion Based on New Approach of Parameters Estimation for Polynomial Phase Signal
}

\author{
Yong Wang and Yi-Cheng Jiang \\ Research Institute of Electronic Engineering Technology, Harbin Institute of Technology, Harbin 150001, China \\ Correspondence should be addressed to Yong Wang, wangyong6012@hit.edu.cn
}

Received 25 September 2010; Revised 20 January 2011; Accepted 9 March 2011

Academic Editor: M. Greco

Copyright () 2011 Y. Wang and Y.-C. Jiang. This is an open access article distributed under the Creative Commons Attribution License, which permits unrestricted use, distribution, and reproduction in any medium, provided the original work is properly cited.

ISAR imaging of ships at sea with significant motion results in the Doppler frequency shift for the received signal is time-varying, which will deteriorate the ISAR image quality for the Range-Doppler (RD) algorithm. In this paper, the received signal is modeled as a multicomponent cubic phase signal (CPS), and a new method for estimating the parameters of CPS based on the integrated high-order matched phase transform (IHMPT) is proposed. This algorithm is simpler and more computational efficient than some of other parameters estimation algorithms proposed previously. Then, combined with the Range-Instantaneous-Doppler (RID) technique, the high quality instantaneous ISAR images can be obtained. The results of simulated and measured data are provided to demonstrate the effectiveness of the new method proposed.

\section{Introduction}

The Inverse Synthetic Aperture Radar (ISAR) technique has attracted the attention of many scholars all around the world, and many useful results have been obtained in the past two decades [1-4], especially for the ISAR imaging of plane target. The ISAR imaging of ship target is also very important in the national defense, such as the target recognition and battlefield awareness $[5,6]$. The imaging condition for ship target is more complicated than the plane due to the extreme sea environment. This sea-induced motion results in chaotic, complex, three-dimensional (3D) motion and does not conform to the ISAR imaging assumptions of planar, constant rate rotation. In this case, the Doppler frequency shift for the received signal is time-varying, which will deteriorate the ISAR image quality for the Range-Doppler (RD) algorithm.

The Range-Instantaneous-Doppler (RID) algorithm was presented for ISAR imaging of maneuvering target in [710], where the Doppler analysis for $\mathrm{RD}$ algorithm is replaced by the time frequency analysis, such as the DechirpClean method [11], the Radon-Wigner transform [12], and the adaptive Chirplet decomposition algorithm [13]. These algorithms are based on the assumption that the received signal in a range bin is a multicomponent linear-frequencymodulated (LFM) signal, and the instantaneous ISAR images can be obtained by estimating the parameters of it. Hence, these algorithms are suitable in situations where the maneuverability is not too severe. For the ship target with high maneuverability, the algorithms presented in [11-13] will not be appropriate for the sake of high-order phase term in the received signal. In $[14,15]$, the received signal is modeled as multicomponent cubic phase signal for the ship target, and a TC-DechirpClean algorithm was presented to estimate the parameters of it. But this algorithm requires a two-dimensional maximization to estimate the parameters of the cubic phase signal, and it therefore suffers from a high computational load. In [16], the product high-order matched-phase transform (PHMT) is proposed for ISAR imaging of ship target by the authors. By the multiplication of high-order matched-phase transform slices for the cubic phase signal at different time positions, the parameters of the multicomponent cubic phase signal can be estimated. But the selection and total number of time positions will influence the parameters estimation accuracy. In $[17,18]$, the polynomial Fourier transform and local polynomial Fourier transform are used in ISAR or SAR imaging, but these algorithms require significant memory and calculations. 


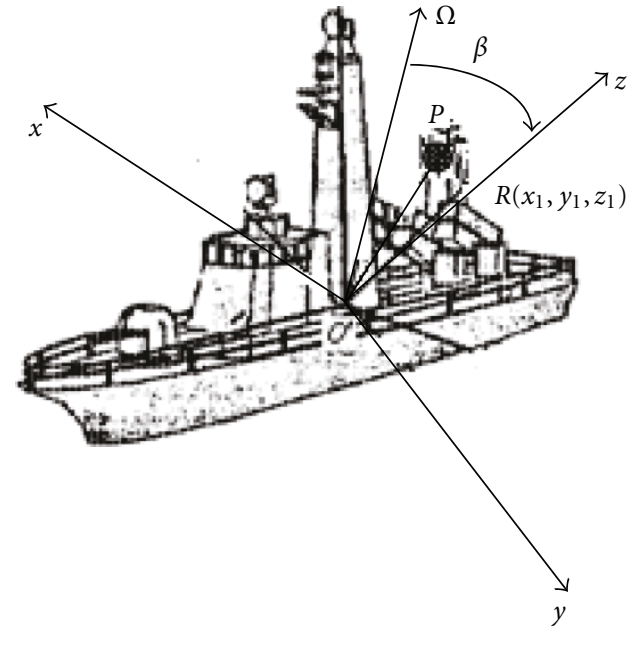

FIGURE 1: Geometry of ISAR image for ship target.

In this paper, the received signal is modeled as multicomponent cubic phase signal (CPS), and the integrated high-order matched phase transform (IHMPT) is presented to estimate the parameters of it. This method requires only one-dimensional maximization, and the parameters of each component can be estimated efficiently. Then, the high-quality instantaneous ISAR images can be obtained combined with the RID technique.

This paper is organized as follows. In Section 2, the cubic phase signal model for the received signal of ship target in ISAR imaging is established; in Section 3, the principle of IHMPT is presented, and the ISAR imaging algorithm of ship target based on the IHMPT is discussed in Section 4; the results for simulated and measured data are given in Section 5; Section 6 is the conclusion for the paper.

\section{Cubic Phase Signal Model for the Received Signal}

In this section, we assume that the motion compensation (including the range alignment and phase adjustment) has been completed, and the ISAR imaging geometry for ship target is shown in Figure 1.

In Figure 1, the coordinate of the radar line-of-sight (LOS) is $x y z$ and the synthetic vector $\boldsymbol{\Omega}$ denotes the angular velocity of the target. The $x$ axis is located on the $z-\Omega$ plane, and the unit vector of LOS is $\mathbf{r}$, which overlaps the $z$ axis. The point $O$ is the rotating centre of the ship target, and we use the vector $\mathbf{R}\left(x_{1}, y_{1}, z_{1}\right)$ to denote the position of a scatterer $P$ on the target. The Doppler frequency shift of the scatterer induced by the rotation can be written as [8]

$$
\omega=\frac{4 \pi}{\lambda}[(\boldsymbol{\Omega} \times \mathbf{R}) \bullet \mathbf{r}]
$$

where $\lambda$ is the wavelength of the radar, $\times$ denotes the outer product, and $\bullet$ denotes the inner product.
For ship target with high maneuverability, the synthetic vector $\Omega$ can be approximated as follows:

$$
\mathbf{\Omega} \approx \mathbf{\Omega}_{0}+\boldsymbol{\alpha}_{0} t+\gamma_{0} t^{2},
$$

where $\boldsymbol{\Omega}_{0}, \boldsymbol{\alpha}_{0}$, and $\boldsymbol{\gamma}_{0}$ are the constant term, first-order term, and second-order term coefficients of $\boldsymbol{\Omega}$, respectively. $t$ is the azimuth time. Then, we can rewrite (1) as follows:

$$
\begin{aligned}
\omega & =\frac{4 \pi}{\lambda}[(\boldsymbol{\Omega} \times \mathbf{R}) \bullet \mathbf{r}]=\frac{4 \pi}{\lambda}[\boldsymbol{\Omega} \bullet(\mathbf{R} \times \mathbf{r})] \\
& =\frac{4 \pi}{\lambda}\left[\boldsymbol{\Omega}_{0} \bullet \boldsymbol{\mu}+\boldsymbol{\alpha}_{0} \bullet \boldsymbol{\mu} t+\gamma_{0} \bullet \boldsymbol{\mu} t^{2}\right]
\end{aligned}
$$

where $\boldsymbol{\mu}=\mathbf{R} \times \mathbf{r}$. Then, for the Doppler frequency $\omega$, the received signal for scatterer $P$ can be written as

$$
\begin{aligned}
s_{0}(t) & =A_{0} \exp (j \omega t) \\
& =A_{0} \exp \left[j \frac{4 \pi}{\lambda}\left(\boldsymbol{\Omega}_{0} \bullet \boldsymbol{\mu} t+\boldsymbol{\alpha}_{0} \bullet \boldsymbol{\mu} t^{2}+\boldsymbol{\gamma}_{0} \bullet \boldsymbol{\mu} t^{3}\right)\right],
\end{aligned}
$$

where $A_{0}$ is the amplitude. From (4), we can see that for a scatterer on the ship target, the received signal has the form of cubic phase signal (CPS). Hence, for multiple scatterers in a certain range bin, the received signal can be characterized as

$$
\begin{aligned}
s(t) & =\sum_{k=1}^{K} A_{k} \exp \left[j \frac{4 \pi}{\lambda}\left(\boldsymbol{\Omega}_{0 k} \bullet \boldsymbol{\mu}_{k} t+\boldsymbol{\alpha}_{0 k} \bullet \boldsymbol{\mu}_{k} t^{2}+\boldsymbol{\gamma}_{0 k} \bullet \boldsymbol{\mu}_{k} t^{3}\right)\right] \\
& =\sum_{k=1}^{K} A_{k} \exp \left[j\left(a_{k, 1} t+a_{k, 2} t^{2}+a_{k, 3} t^{3}\right)\right],
\end{aligned}
$$

where

$$
\begin{aligned}
& a_{k, 1}=\left(\frac{4 \pi}{\lambda}\right) \boldsymbol{\Omega}_{0 k} \bullet \boldsymbol{\mu}_{k}, \\
& a_{k, 2}=\left(\frac{4 \pi}{\lambda}\right) \boldsymbol{\alpha}_{0 k} \bullet \boldsymbol{\mu}_{k}, \\
& a_{k, 3}=\left(\frac{4 \pi}{\lambda}\right) \gamma_{0 k} \bullet \boldsymbol{\mu}_{k},
\end{aligned}
$$

and $K$ is the number of scatterers in a range bin, $A_{k}(k=$ $1,2, \ldots K)$ is the amplitude of each scatterer, and $\left.a_{k, l}\right|_{l=1} ^{3}$ are the phase coefficients to be determined.

It can be seen from (5) that the received signal in a range bin can be modeled as multicomponent CPS for ISAR imaging of ship target with high maneuverability. In this paper, a new algorithm called IHMPT is proposed to estimate the parameters of the multicomponent CPS. Combined with the RID algorithm, the high-quality instantaneous ISAR images can be obtained. 


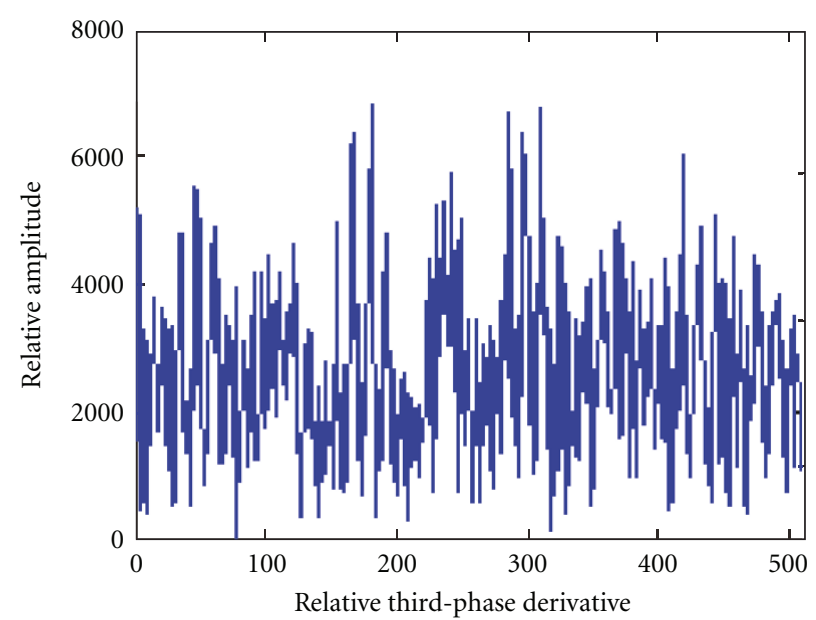

(a) HMPT slice at $n=-55$

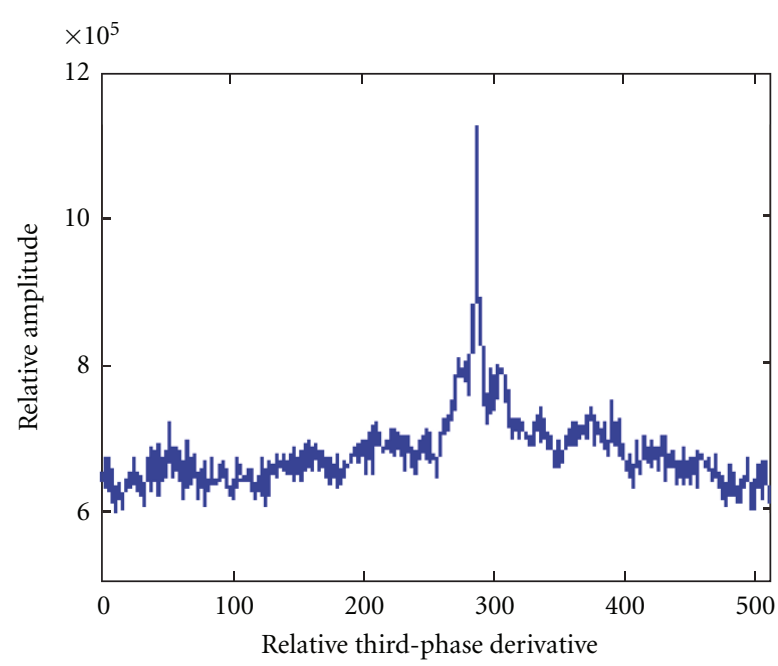

(b) IHMPT for the signal

Figure 2: Results of the numerical example.

\section{Principle of IHMPT}

3.1. The High-Order Matched Phase Transform (HMPT). Consider the discrete form of monocomponent CPS with the following structure:

$$
\begin{gathered}
s(n)=A_{0} \exp \left[j\left(a_{1} n+a_{2} n^{2}+a_{3} n^{3}\right)\right], \\
-\frac{(N-1)}{2} \leq n \leq \frac{(N-1)}{2},
\end{gathered}
$$

where $N$ is the length of the signal, and it is assumed to be an odd integer, $A_{0}$ is the amplitude, and $a_{1}, a_{2}, a_{3}$ are the coefficients to be determined.

The high-order matched phase transform (HMPT) for $s(n)$ was proposed in [16] as follows:

$\operatorname{HMPT}(n, \sigma)$

$$
\begin{aligned}
=\sum_{m=0}^{(N-1) / 2}\left\{\left[s^{*}(n+m) s(n-m)\right]^{2}\left[s(n+2 m) s^{*}(n-2 m)\right]\right\} \\
\quad \times \exp \left(-j \sigma m^{3}\right) .
\end{aligned}
$$

Substitute (7) into (8), we obtain

$$
\operatorname{HMPT}(n, \sigma)=A_{0}^{6} \sum_{m=0}^{(N-1) / 2} \exp \left[j\left(12 a_{3}-\sigma\right) m^{3}\right]
$$

It is obvious that HMPT is independent on $n$ without the consideration of noise. This means that in the $(n, \sigma)$ plane, $\operatorname{HMPT}(n, \sigma)$ is a line parallels to the $n$ axis. curve

We can see from (9) that $|\operatorname{HMPT}(n, \sigma)|$ peaks along the

$$
\sigma=12 a_{3}
$$

Hence, the $a_{3}$ can be estimated by the maximum value of $|\operatorname{HMPT}(n, \sigma)|$ as

$$
\hat{a}_{3}=\arg \max _{\sigma} \frac{|\operatorname{HMPT}(n, \sigma)|}{12} .
$$

Then, the other parameters can be estimated by the dechirp technique and the Fourier transform.

3.2. The Definition of IHMPT. From (8), we can see that the HMPT has the nonlinearity character. Hence, for multicomponent CPS, the cross-terms will appear. In this paper, the IHMPT is proposed to reduce the cross-terms between different components. The definition of IHMPT is

$$
\operatorname{IHMPT}(\sigma)=\sum_{n}|\operatorname{HMPT}(n, \sigma)| .
$$

For the IHMPT, the cross-terms can be reduced due to the dispersion in the $\operatorname{HMPT}(n, \sigma)$ domain, and the autoterms can be amplified due to the integration operation. Hence, the IHMPT is appropriate for the parameters estimation of multicomponent CPS. Furthermore, from the analysis above, we can see that the IHMPT requires only onedimensional maximization, and it is computational efficient, which is quite suitable in ISAR imaging.

3.3. Numerical Example. In this section, we use the numerical example to demonstrate the effectiveness of IHMPT in the suppression of cross-terms for multicomponent CPS. For convenience, we assume that the simulated signal consists of two components with the following structure:

$$
s(n)=\sum_{k=1}^{2} A_{k} \exp \left[j\left(a_{k, 1} n+a_{k, 2} n^{2}+a_{k, 3} n^{3}\right)\right] .
$$

The sampling rate is assumed to be unity and $n \in$ $[-255,255]$. The parameters are shown in Table 1. (In order 


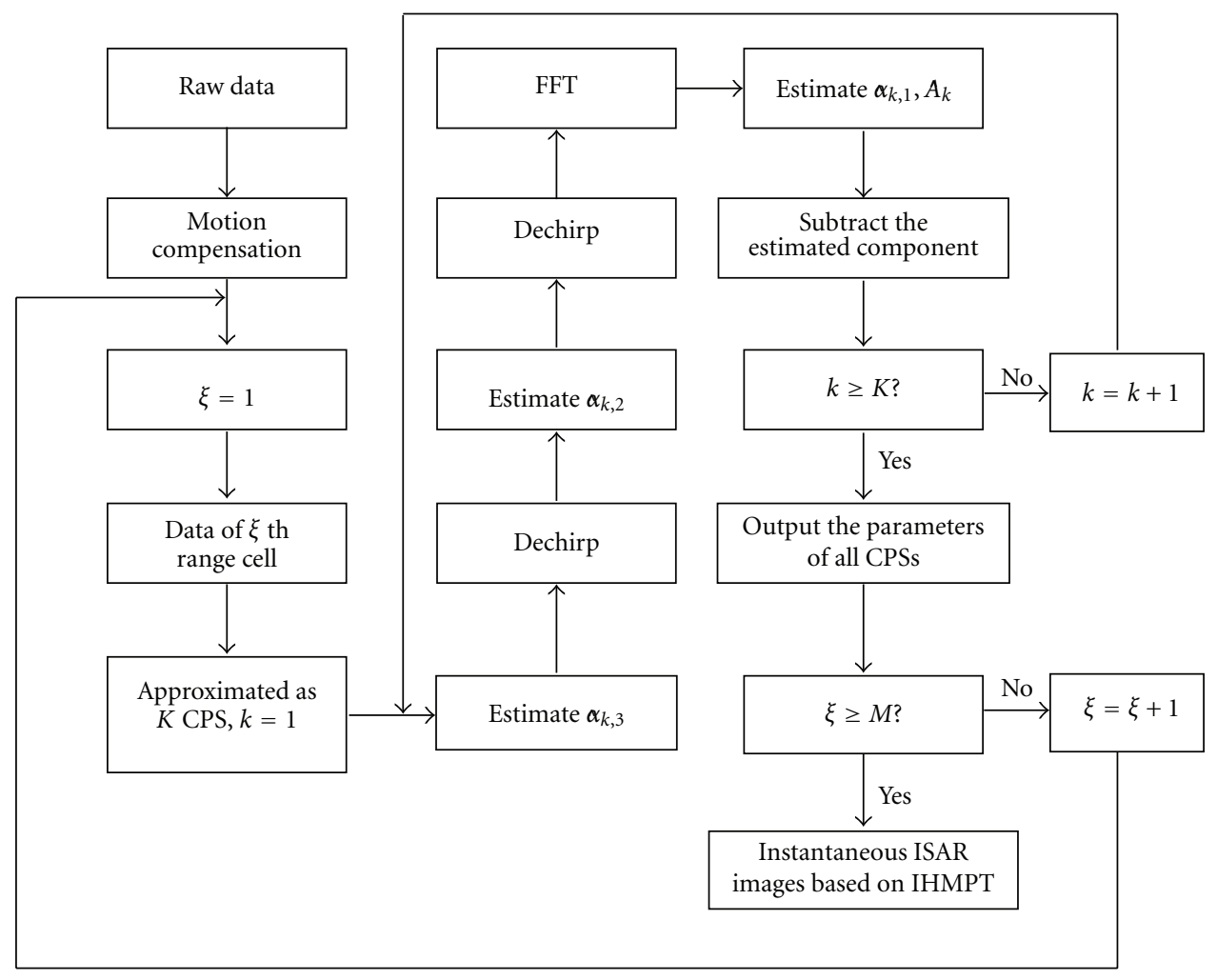

FIGURE 3: Flow chart of ISAR imaging based on IHMPT algorithm.

TABLe 1: Parameters of the simulated signal.

\begin{tabular}{lcccc}
\hline Components $(k)$ & $A_{k}$ & $a_{k, 1}$ & $a_{k, 2}$ & $a_{k, 3}$ \\
\hline 1 & 2 & $\pi / 8$ & $5 \times 10^{-3}$ & $1 \times 10^{-5}$ \\
2 & 1.5 & $\pi / 4$ & $-1 \times 10^{-3}$ & $-6 \times 10^{-5}$ \\
\hline
\end{tabular}

to avoid ambiguities arising from the cyclic nature of spectral transforms of sampled signals [19], it is assumed that $\left|a_{k, i}\right| \leq$ $\pi /\left(i !(N / 2)^{(i-1)}\right), i=1,2,3 . N$ is the length of the signal.)

Figure 2(a) is the HMPT slice at $n=-55$. We can see that the cross-terms appear for the nonlinearity of HMPT, and the auto-terms cannot be detected correctly. Figure 2(b) is the IHMPT for the signal. It is obvious that the crossterms have been suppressed greatly. At the same time, the auto-terms have been amplified greatly, which is appropriate for the parameters estimation of multicomponent CPS. The reason for Figure 2(b) showing one peak is that the amplitudes for the two components are different: one is 2 and the other is 1.5, which is shown in Table 1. This peak denotes the $a_{3}$ parameter for the component with amplitude 2, and after this component is subtracted from the original signal, the other peak for the $a_{3}$ parameter for the component with amplitude 1.5 will appear.

The results for the example have demonstrated the validity of the IHMPT.

\section{ISAR Imaging of Ship Target Based on IHMPT}

The ISAR imaging algorithm of ship target with high maneuverability can be illustrated as follows.

Step 1. Suppose the received signal in a range bin is $K$ components CPS of the discrete form

$$
\begin{gathered}
s(n)=\sum_{k=1}^{K} A_{k} \exp \left[j\left(a_{k, 1} n+a_{k, 2} n^{2}+a_{k, 3} n^{3}\right)\right], \\
-\frac{(N-1)}{2} \leq n \leq \frac{(N-1)}{2},
\end{gathered}
$$

where $A_{k}$ is the amplitude of $k$ th component and $\left.a_{k, l}\right|_{l=1} ^{3}$ is the $l$ th-order phase coefficients for the $k$ th component.

Step 2. Initialize $k=1, s_{1}(n)=s(n)$.

Step 3. Estimate $\hat{a}_{k, 3}$ by finding the peak of $\operatorname{IHMPT}(\sigma)$.

Step 4. Construct the reference signal

$$
s_{\text {refl }}(n)=\exp \left(-j \hat{a}_{k, 3} n^{3}\right)
$$

and multiply it with the signal $s_{k}(n)$; we obtain

$$
s_{d}(n)=s_{k}(n) \cdot s_{\mathrm{ref} 1}(n)=s_{k}(n) \exp \left(-j \hat{a}_{k, 3} n^{3}\right) .
$$




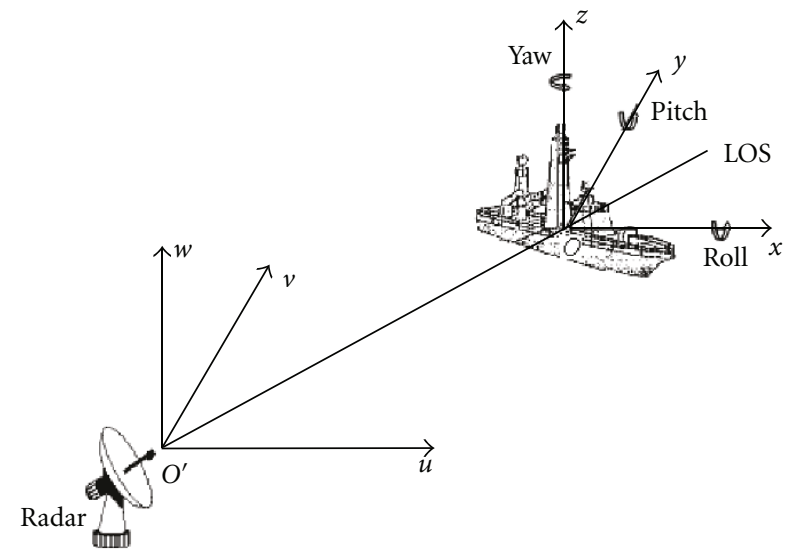

FIgURE 4: Coordinate systems of Radar and ship target.

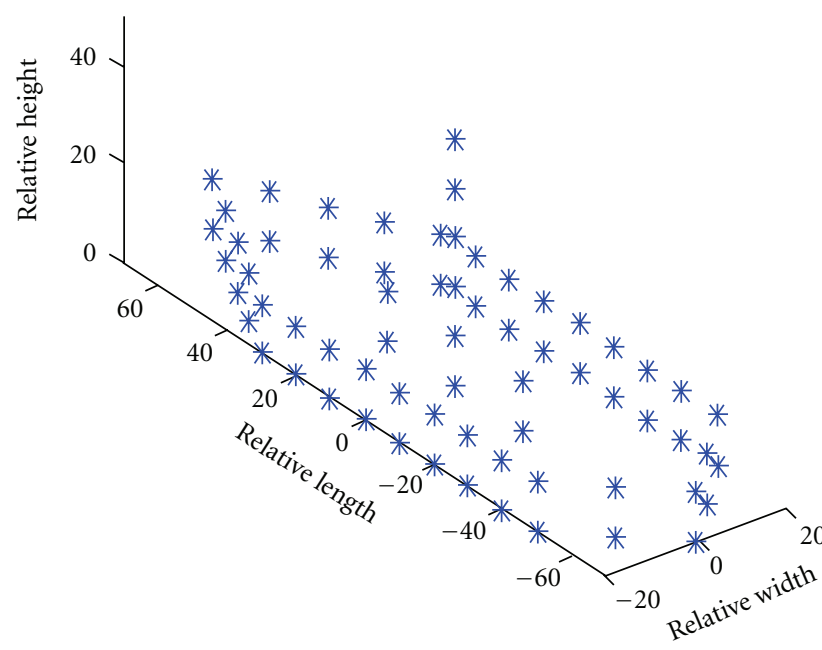

Figure 5: Simulated ship model.

Step 5. Estimate $\hat{a}_{k, 2}$ by the cubic phase function presented as follows:

$\hat{a}_{k, 2}=\frac{\arg \max _{\xi}\left|\sum_{m=0}^{(N-1) / 2} s_{d}(n+m) s_{d}(n-m) \exp \left(-j \xi m^{2}\right)\right|}{2}$.

Step 6. Construct the reference signal

$$
s_{\mathrm{ref} 2}(n)=\exp \left(-j \hat{a}_{k, 3} n^{3}-j \widehat{a}_{k, 2} n^{2}\right) .
$$

Then, estimate $\hat{a}_{k, 1}$ by dechirping the original signal with $s_{\text {ref2 }}(n)$ and finding the Fourier transform peak:

$$
\hat{a}_{k, 1}=\arg \max _{a_{k, 1}}\left|\sum_{n=-(N-1) / 2}^{(N-1) / 2} s_{k}(n) \cdot s_{\mathrm{ref} 2}(n) \cdot \exp \left(-j a_{k, 1} n\right)\right| .
$$

TABLE 2: Simulation parameters.

\begin{tabular}{lcc}
\hline & Amplitude $\left(^{\circ}\right)$ & Angular velocity (radian/s) \\
\hline Roll & 15 & $2 \pi / 14$ \\
Pitch & 4 & $2 \pi / 7$ \\
Yaw & 2 & $2 \pi / 14$ \\
\hline
\end{tabular}

Step 7. Estimate $\hat{A}_{k}$ as follows:

$$
\widehat{A}_{k}=\left|\frac{1}{N} \sum_{n=-(N-1) / 2}^{(N-1) / 2} s_{k}(n) e^{-j\left(\hat{a}_{k, 1} n+\hat{a}_{k, 2} n^{2}+\hat{a}_{k, 3} n^{3}\right)}\right| .
$$

Step 8. Subtract the estimated $k$ th component from $s_{k}(n)$ :

$$
s_{k+1}(n)=s_{k}(n)-\hat{A}_{k} e^{j\left(\hat{a}_{k, 1} n+\hat{a}_{k, 2} n^{2}+\hat{a}_{k, 3} n^{3}\right)} .
$$

Step 9. Set $k=k+1$, and repeat the above steps until $k=$ $K$ or the residual energy of the signal is less than a threshold $\varepsilon$ (example, $1 \%$ of the original signal).

Based on the above procedure, we can obtain the instantaneous ISAR image at different time positions based on the IHMPT, which is illustrated in Figure 3. The number of time history series is $P$, and each has the length of $M$. After computing the IHMPT of each range bin and time sampling, the $P$ frames $M \times P$ instantaneous ISAR images can be obtained.

\section{Examples}

In this section, the results of simulated and measured data are provided to demonstrate the effectiveness of the IHMPT algorithm for ISAR imaging of ship target with high maneuverability.

5.1. Simulated Data. Here, we use the simulated data of ship target with three-dimensional rotation (including the roll, pitch, and yaw) to demonstrate the effectiveness of the IHMPT algorithm.

The coordinate systems of Radar and the ship target are shown in Figure 4, where the $(u, v, w)$ frame is defined as the Radar coordinate frame and the $(x, y, z)$ frame is defined as the target coordinate frame. We assume that the Radar is located at the origin $O^{\prime}$ of the $(u, v, w)$ coordinate, and the initial location of the rotating centre of the ship target $O$ in the $(u, v, w)$ coordinate is $\left(u_{0}, v_{0}, w_{0}\right)$. The direction for the axis $x, y$, and $z$ is parallel to the axis $u, v$, and $w$.

The instantaneous angular position of the target for the yaw, roll, and pitch motion can be described as follows [15]:

$$
\begin{aligned}
& \theta_{r}(t)=q_{r} \sin \left(\omega_{r} t\right), \\
& \theta_{p}(t)=q_{p} \sin \left(\omega_{p} t\right), \\
& \theta_{y}(t)=q_{y} \sin \left(\omega_{y} t\right),
\end{aligned}
$$




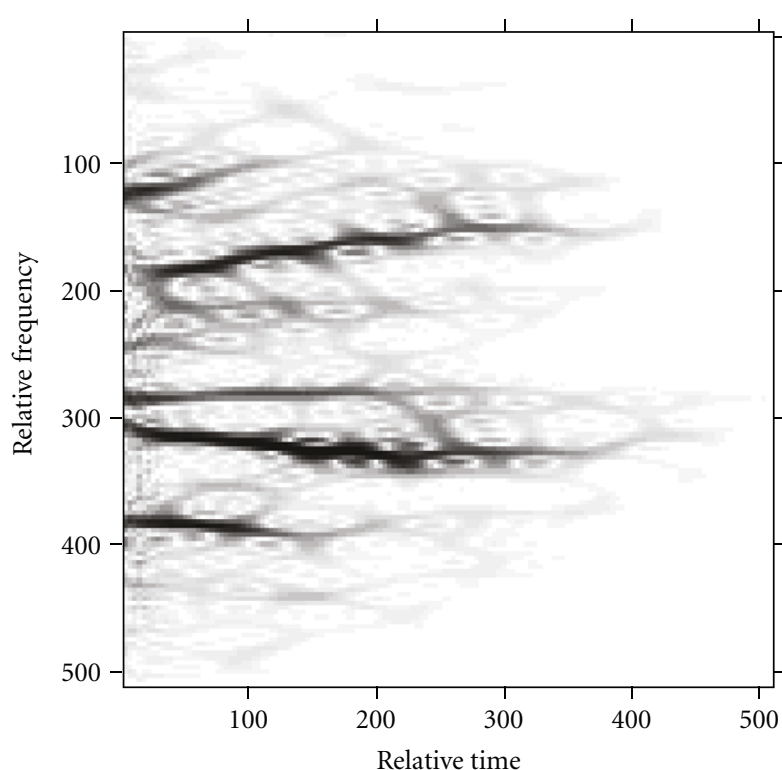

(a) 1220th range bin

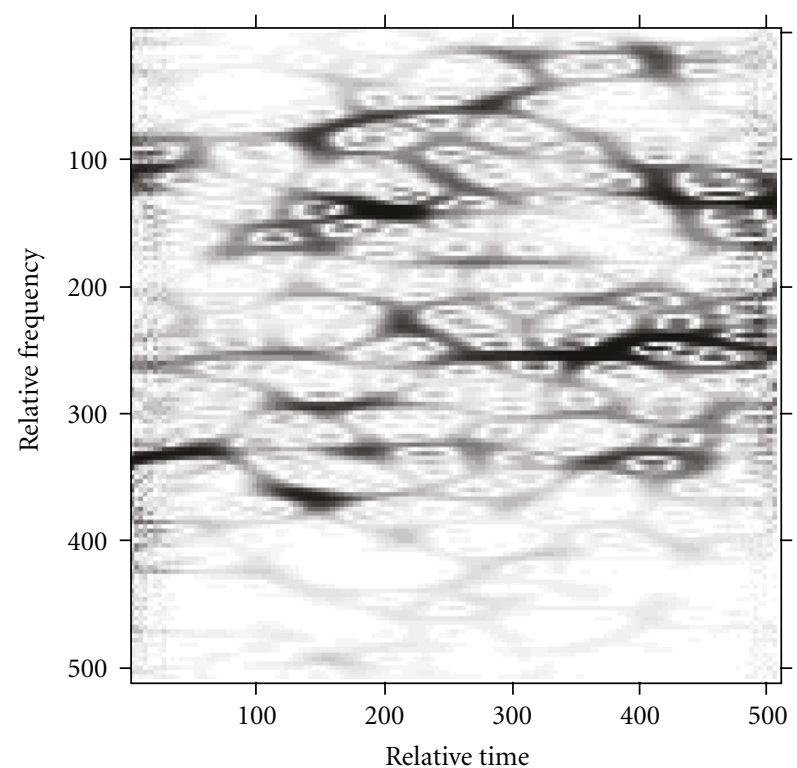

(b) 1251th range bin

FIGURE 6: SPWVD of the received signal in a range bin.

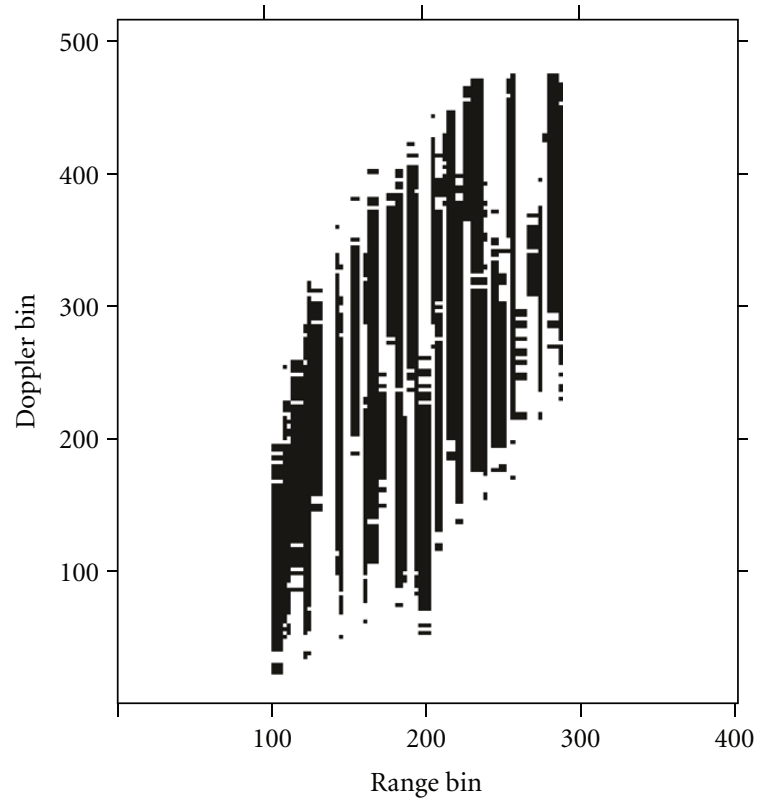

FIGURE 7: ISAR image of ship target based on the RD algorithm.

TABle 3: Parameters for the simulated data.

\begin{tabular}{lccc}
\hline Bandwidth & Carrier frequency & Pulse width & Sampling frequency \\
$B=400 \mathrm{MHz}$ & $f_{c}=10 \mathrm{GHz}$ & $T_{p}=20 \mu \mathrm{s}$ & $f_{s}=120 \mathrm{MHz}$ \\
\hline Sampling number & Pulse repetition frequency & Number of pulses & Number of scatterers \\
$N=2400$ & $\mathrm{PRF}=625 \mathrm{~Hz}$ & 1024 & 66 \\
\hline Translational velocity of ship & The angle between the velocity and the $u$ axis & The initial location of the ship target in $(u, v, w)$ coordinate \\
$V=20.5778 \mathrm{~m} / \mathrm{s}$ & \multicolumn{2}{c}{$\pi / 3$} & $u_{0}=1000, v_{0}=1000, w_{0}=-300$ \\
\hline
\end{tabular}




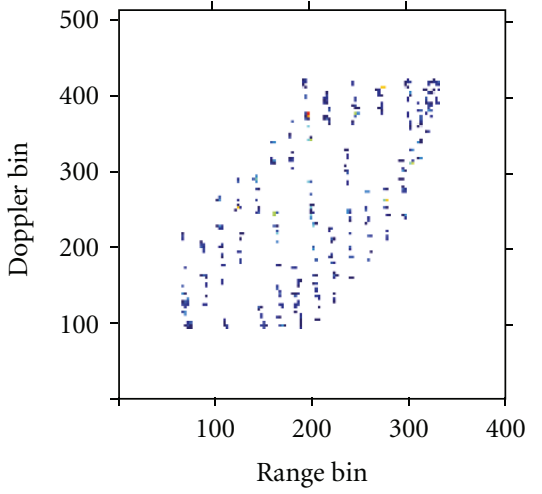

(a)

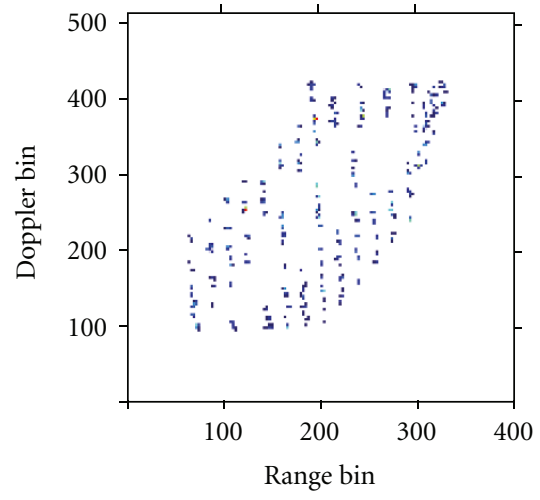

(b)

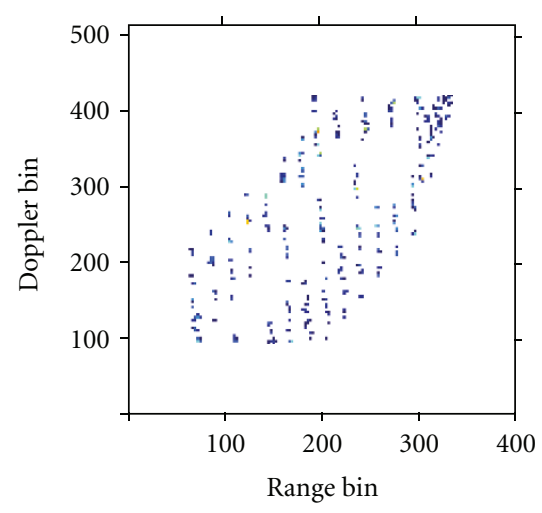

(c)

FIGURE 8: Instantaneous ISAR images based on the IHMPT.

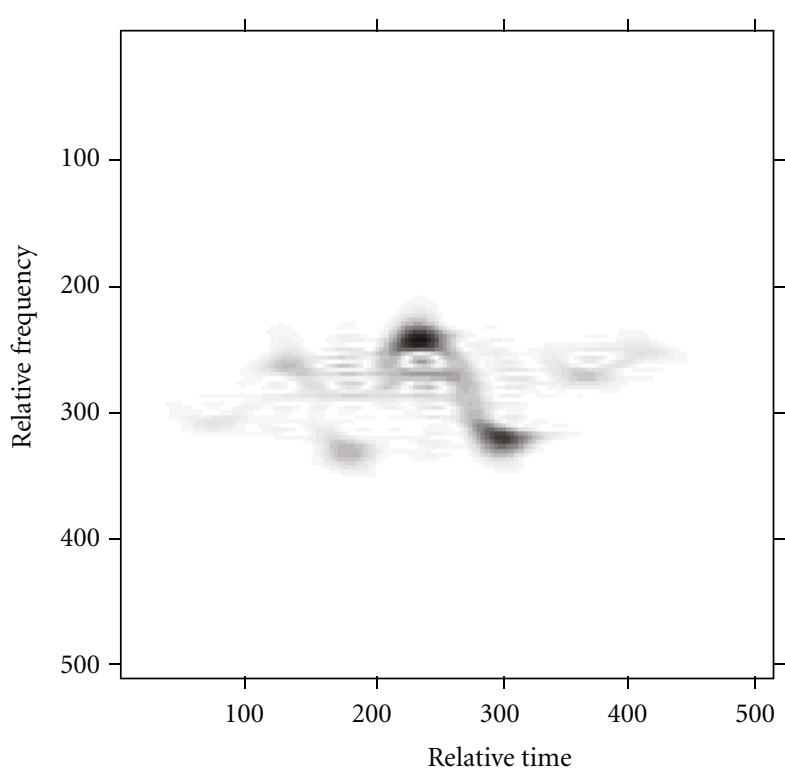

(a) 610th range bin

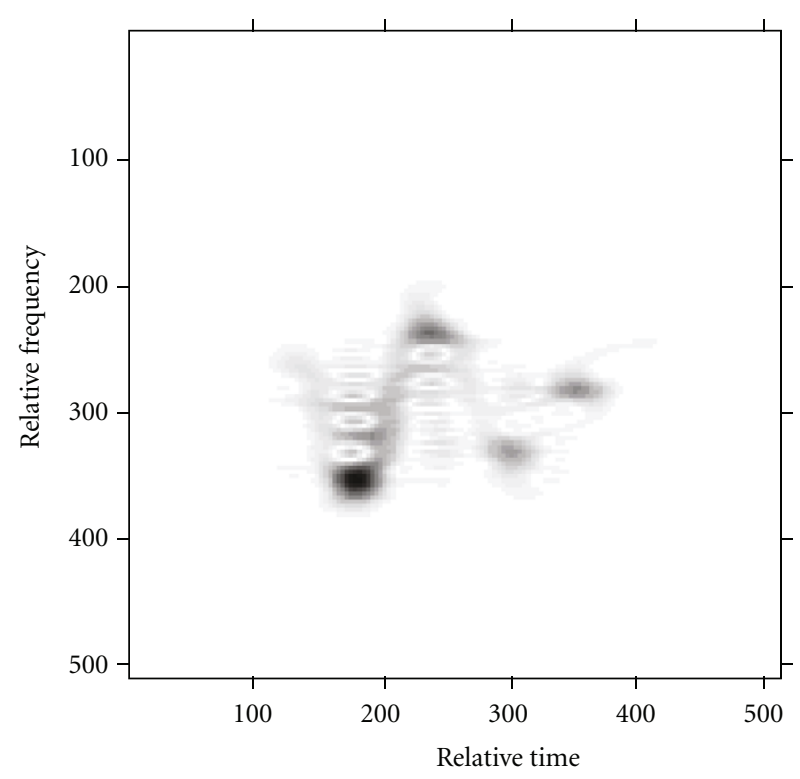

(b) 620th range bin

FIGURE 9: SPWVD of the received signal in a range bin.

where $q_{r}, q_{p}$, and $q_{y}$ are the angular amplitudes in radians and $\omega_{r}, \omega_{p}$, and $\omega_{y}$ are the roll, pitch, and yaw angular velocities, respectively.

The rotation parameters of the target are shown in Table 2, and the other parameters for the simulated data are shown in Table 3.

The three-dimensional (3D) image of the target is shown in Figure 5.

We choose the received signal of the 1220th and 1251th range bins, and compute the smoothed pseudo-Wigner-Ville distribution (SPWVD) of them, which are shown in Figure 6.

From Figure 6, we can see that the time-varying character for the Doppler frequency is very complicated, and this demonstrates that the ship target has high maneuverability.
Figure 7 is the ISAR image based on the RD algorithm. For the high maneuverability of the target, the image is blurred severely.

Figure 8 shows the instantaneous ISAR images at different time positions based on the IHMPT algorithm; it is obvious that the image quality has been improved greatly.

5.2. Measured Data. We choose a set of measured data for ship target to demonstrate the effectiveness of the IHMPT. The radar works in the X band, and the radar parameters are not authorized to be published. The SPWVDs for the received signal in the 610th range bin and 620th range bin are shown in Figure 9; it is obvious that the ship target has high maneuverability. 


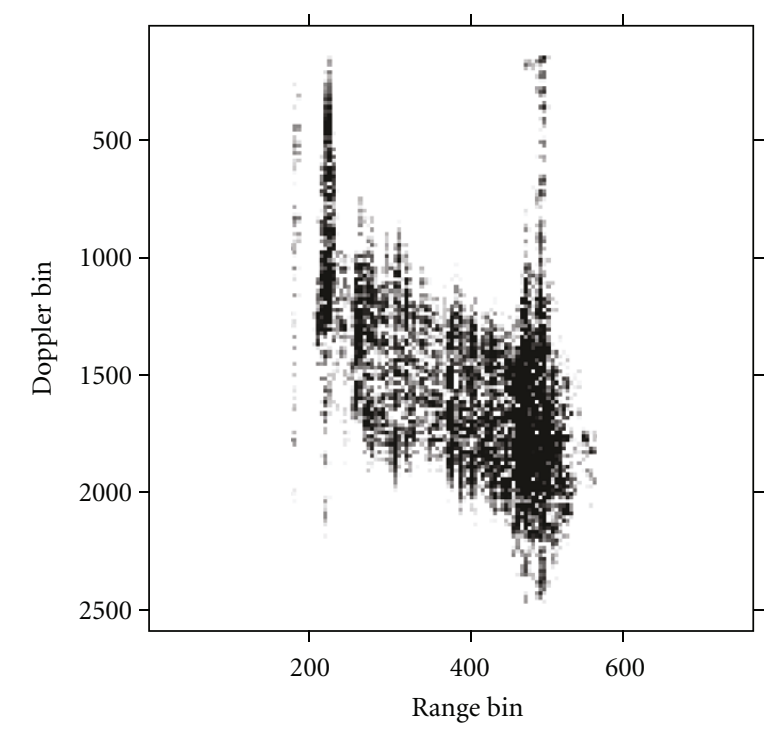

FIGURE 10: ISAR image of ship target based on the RD algorithm.

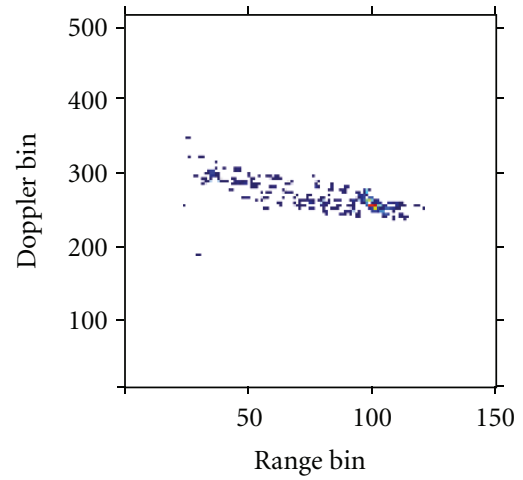

(a)

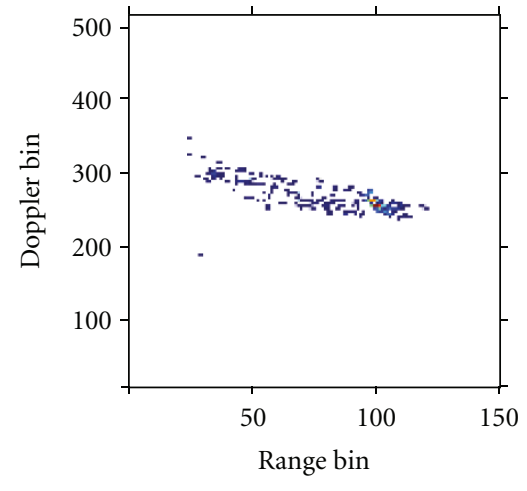

(b)

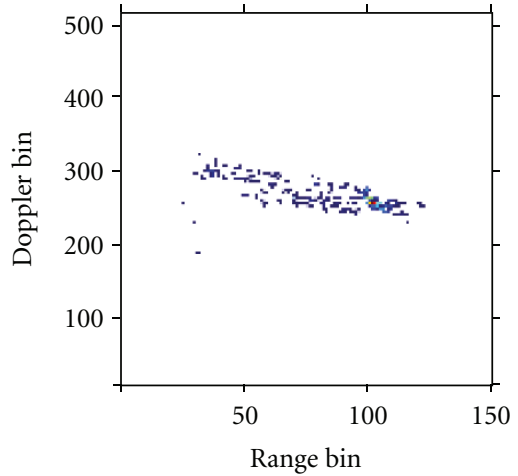

(c)

FIGURE 11: Instantaneous ISAR images based on the IHMPT.

Figure 10 is the ISAR image based on the RD algorithm. For the high maneuverability of the target, the image is blurred severely.

The instantaneous ISAR images at different time positions based on the IHMPT algorithm are shown in Figure 11. It is obvious that the quality has been improved greatly, which demonstrates the effectiveness of the IHMPT algorithm proposed.

\section{Conclusion}

For ISAR imaging of ship target with high maneuverability, the received signal in a range bin can be modeled as multicomponent cubic phase signal. The IHMPT can be used to estimate the parameters of the multicomponent cubic phase signal, then combined with the RID technique, the high quality instantaneous ISAR images can be obtained.

\section{Acknowledgments}

This work was supported in part by the National Natural Science Foundation of China under Grant no. 61001166, the National Science Foundation for Post-doctoral Scientists of China under Grants nos. 20080440135 and 200902413, the Heilongjiang Postdoctoral Grant no. LBH-Z08114, the Project Supported by Development Program for Outstanding Young Teachers in Harbin Institute of Technology under Grant HITQNJS. 2009. 060, and the Specialized Research Fund for the Doctoral Program of Higher Education under Grant no. 20092302120002. 


\section{References}

[1] M. Martorella, "Novel approach for ISAR image crossrange scaling," IEEE Transactions on Aerospace and Electronic Systems, vol. 44, no. 1, pp. 281-294, 2008.

[2] T. Thayaparan, L. J. Stankovic, C. Wernik, and M. Dakovic, "Real-time motion compensation, image formation and image enhancement of moving targets in ISAR and SAR using S-method-based approach," IET Signal Processing, vol. 2, no. 3, pp. 247-264, 2008.

[3] M. Martorella and F. Berizzi, "Time windowing for highly focused ISAR image reconstruction," IEEE Transactions on Aerospace and Electronic Systems, vol. 41, no. 3, pp. 992-1007, 2005.

[4] V. C. Chen and S. Qian, "Joint time-frequency transform for radar range-doppler imaging," IEEE Transactions on Aerospace and Electronic Systems, vol. 34, no. 2, pp. 486-499, 1998.

[5] V. Zeljkovic, Q. Li, R. Vincelette, C. Tameze, and F. Liu, "Automatic algorithm for inverse synthetic aperture radar images recognition and classification," IEE Proceedings Radar, Sonar and Navigation, vol. 4, no. 1, pp. 96-109, 2010.

[6] M. Xing, R. Guo, C. W. Qiu, L. Liu, and Z. Bao, "Experimental research of unsupervised Cameron/maximum-likelihood classification method for fully polarimetric synthetic aperture radar data," IEE Proceedings Radar, Sonar and Navigation, vol. 4, no. 1, pp. 85-95, 2010.

[7] S. K. Wong, G. Duff, and E. Riseborough, "Distortion in the inverse synthetic aperture radar (ISAR) images of a target with time-varying perturbed motion," IEE Proceedings: Radar, Sonar and Navigation, vol. 150, no. 4, pp. 221-227, 2003.

[8] F. Berizzi, E. D. Mese, M. Diani, and M. Martorella, "Highresolution ISAR imaging of maneuvering targets by means of the range instantaneous Doppler technique: modeling and performance analysis," IEEE Transactions on Image Processing, vol. 10, no. 12, pp. 1880-1890, 2001.

[9] T. Thayaparan, G. Lampropoulos, S. K. Wong, and E. Riseborough, "Application of adaptive joint time-frequency algorithm for focusing distorted ISAR images from simulated and measured radar data," IEE Proceedings: Radar, Sonar and Navigation, vol. 150, no. 4, pp. 213-220, 2003.

[10] A. D. Lanterman, D. C. Munson, and Y. Wu, "Wide-angle radar imaging using time-frequency distributions," IEE Proceedings: Radar, Sonar and Navigation, vol. 150, no. 4, pp. 203 211, 2003.

[11] Z. Bao, C. Sun, and M. Xing, "Time-frequency approaches to ISAR imaging of maneuvering targets and their limitations," IEEE Transactions on Aerospace and Electronic Systems, vol. 37, no. 3, pp. 1091-1099, 2001.

[12] G. Wang, Z. Bao, and X. Sun, "Inverse synthetic aperture radar imaging of nonuniformly rotating targets," Optical Engineering, vol. 35, no. 10, pp. 3007-3011, 1996.

[13] J. Li and H. Ling, "Application of adaptive chirplet representation for ISAR feature extraction from targets with rotating parts," IEE Proceedings: Radar, Sonar and Navigation, vol. 150, no. 4, pp. 284-291, 2003.

[14] Y. Li, R. Wu, M. Xing, and Z. Bao, "Inverse synthetic aperture radar imaging of ship target with complex motion," IET Radar, Sonar and Navigation, vol. 2, no. 6, pp. 395-403, 2008.

[15] G. Zhao-Zhao, L. Ya-Chao, X. Meng-Dao, W. Genyuan, Z. Shou-Hong, and B. Zheng, "ISAR imaging of manoeuvring targets with the range instantaneous chirp rate technique," IET Radar, Sonar and Navigation, vol. 3, no. 5, pp. 449-460, 2009.
[16] Y. Wang and Y. Jiang, "ISAR imaging of a ship target using product high-order matched-phase transform," IEEE Geoscience and Remote Sensing Letters, vol. 6, no. 4, Article ID 4785239, pp. 658-661, 2009.

[17] I. Djurovic, T. Thayaparan, and L. J. Stankovic, "Adaptive local polynomial Fourier transform in ISAR," EURASIP Journal on Applied Signal Processing, Article ID 36093, 15 pages, 2006.

[18] I. Djurović, T. Thayaparan, and L. J. Stanković, "SAR imaging of moving targets using polynomial Fourier transform," IET Signal Processing, vol. 2, no. 3, pp. 237-246, 2008.

[19] S. Peleg and B. Friedlander, "Discrete polynomial-phase transform," IEEE Transactions on Signal Processing, vol. 43, no. 8, pp. 1901-1914, 1995. 